\title{
Temporal and cross-range coherence of sound traveling through shallow-water nonlinear internal wave packets
}

\author{
Timothy F. Duda \\ Applied Ocean Physics and Engineering Dept., MS 11, Woods Hole Oceanographic Institution, \\ Woods Hole, Massachusetts 02543
}

(Received 8 September 2005; revised 5 April 2006; accepted 6 April 2006)

\begin{abstract}
Expressions governing coherence scales of sound passing through a moving packet of nonlinear internal waves in a continental shelf environment are presented. The expressions describe the temporal coherence scale at a point, and the horizontal coherence scale in a plane transverse to the acoustic path, respectively. Factors in the expressions are the wave packet propagation speed, wave packet propagation direction, the fractional distance from the packet to the source, and the spatial scale $S$ of packet displacement required to cause acoustic field decorrelation. The scale $S$ is determined by the details of coupled mode propagation within the packet and the waveguide. Here, $S$ is evaluated as a function of frequency for one environment, providing numerical values for the coherence scales of this environment. Coherence scales derived from numerical simulation of coupled mode acoustic propagation through moving wave packets substantiate the expressions. (C) 2006 Acoustical Society of America. [DOI: 10.1121/1.2200699]
\end{abstract}

PACS number(s): 43.30.Re, 43.30.Bp, 43.30.Ft [AIT]

Pages: $3717-3725$

\section{INTRODUCTION}

Recent theoretical, computational, and observational papers have demonstrated that nonlinear internal waves are responsible for fluctuations of sound (of a few hundred hertz) propagating a few tens of kilometers on continental shelves. Research into this problem accelerated after demonstration that anomalous transmission loss between 300 and $1100 \mathrm{~Hz}$, observed in the Yellow Sea, was consistent with coupledmode propagation through packets of nonlinear internal waves (Zhou, Zhang, and Rogers). That work raised the possibility of a resonance effect between the internal wave wavenumber and the difference wavenumber of interacting modes.

A subsequent paper showed that mode coupling within individual solitary waves could be described by distinct coupling events at each of the two sides of the wave (Preisig and Duda, 1997). That paper also showed that the vertical mode shape overlap at depths having strong lateral gradients of sound speed played a strong role in mode coupling, as expected, and demonstrated that waves with small lateral scale produce little coupling, regardless of their amplitude, because of canceling coupling events. A resonance effect between the internal wave horizontal wavenumber and the difference horizontal wavenumber of the incident (coupledfrom) and transmitted (coupled-to) modes was not found to be responsible for strong mode coupling in the particular cases of waveguide geometry and frequency that were chosen for study. Instead, resonance and phasing of dominant modes propagating in the wave center (the trough for a nonlinear wave of depression) were found to be of central importance. This work shows that resonance between the internal wave scale and the mode interference scale, as discussed in Frank et al. (2004) and papers cited therein, must play a role, but that the mode pair or pairs responsible for the largest effects may be difficult to ascertain.
Further work showed that the mode coupling process is sensitive to the precise location of internal waves or wave packets (Duda and Preisig, 1999). This sensitivity to position, coupled with the propagating nature of ocean internal waves, creates a time dependence in the resultant acoustic fields. The effect is caused by the changing interrelations of model phases at the point where sound encounters a moving wave. Intuitively, this leads to the temporal decorrelation of signals transmitted from a fixed source to a fixed receiver that pass through moving internal waves (Duda and Preisig, 1999; Rouseff et al., 2002). To apply a nomenclature to this process, let $S$ (with $S>0$ ) be the internal wave (or packet) horizontal displacement scale along the acoustic propagation path that leads to a typical intensity or phase fluctuation at the receiver. That is, movement of the wave of distance $S$ causes an acoustic fluctuation of a typical magnitude.

In the field, measurements of intensity time series and of temporal correlation functions, collected using fixed acoustic propagation paths containing moving internal waves, exhibit fluctuating behavior. Particular measured examples are the rapid swings in signal energy arriving at vertical line arrays over intervals of only a few minutes (Duda et al., 2004; Duda and Preisig, 1999; Lynch et al., 2003; Chiu et al., 2004). Here, by the term signal energy we mean depthintegrated intensity, which is used in other papers on this topic (Duda and Preisig, 1999; Oba and Finette, 2002). Many aspects of the fluctuations essentially validate that wave motion causes the fluctuations (i.e., that moving waves are responsible for fluctuations and that the scale $S$ exists). It is possible that $S$ is not constant at any single location, changing, for example, over a fortnightly tidal cycle as nonlinear wave heights change. However, if the change is slow, then it is reasonable to model the process with a quasisteady scale $S$.

To further examine aspects of wave-induced acoustic fluctuations, two expressions are derived in this paper for the idealized situation of propagation through a single packet of 
internal waves with long straight crests to a horizontal line of receivers. The first expression is for the temporal coherence scale $T_{c}$ of the received signal as a function of three parameters: $S$, the wave (packet) speed, and the wave (packet) direction. This is little more than a definition, and stems directly from the existence of the scale $S$. The other is an expression for the horizontal signal coherence scale $Y_{c}$ in a plane normal to the acoustic path, which is written as a function of four parameters: $S$, source receiver distance $R$, source to packet distance $R_{P}$, and the wave packet angle with respect to the acoustic path. As in previous work (Duda, 2004; Duda and Preisig, 1999; Preisig and Duda, 1997), sound propagating distances of order $o(30) \mathrm{km}$ in a waveguide with $o(100) \mathrm{m}$ water depth is considered.

The ultimate usefulness of the expressions may lie in the possibility of predicting one of the three coherence scales $S, T_{c}$, and $Y_{c}$ after measuring another of them while simultaneously knowing properties of the internal waves. For example, signal energy temporal coherence scale measurements at a vertical line array, when combined with internal wave angle and phase speed measurements (as from satellite synthetic aperture radar), may yield the horizontal crossrange coherence scale.

Results of the expressions are compared with coherence functions computed using $N$-by-two-dimensional $(N \times 2$-D) simulations of three-dimensional acoustic propagation, where $N$ is the number of azimuthal planes passing through the source for which outgoing sound is modeled using a parabolic wave equation. $N \times 2$-D simulations are sufficient for the situation of negligible azimuthal scattering, which has been found to be the case for sound traveling at angles from $0^{\circ}$ to $70^{\circ}$ with respect to the propagation direction of longcrested internal waves (Oba and Finette, 2002). Thus, we address $70^{\circ}$ of the possible $90^{\circ}$ of the angle between the wave crests and the acoustic path. This is the regime where mode coupling dominates over mode refraction. Threedimensional acoustic effects, such as mode refraction, may occur at the remaining $20^{\circ}$ and are covered in other papers (Badiey et al., 2005).

Other water-column processes and seafloor structures may also cause spatial and temporal decorrelation. When this occurs, the results shown here for decorrelation caused by a single packet of waves between the source and receiver may represent upper bounds on correlation distances and times. In situations where a single packet is the primary cause of acoustic fluctuation, the results might allow a useful prediction of correlation scales.

The paper is organized as follows. The geometry of the problem is defined and the coherence scale expressions are developed in Sec. II. In Sec. III, coherence scale results from the expressions are compared with computational simulation results. In Sec. IV, the scale $S$, an important factor governing coherence scales $T_{c}$ and $Y_{c}$, is evaluated as a function of frequency. In Sec. V we discuss nondimensional cross-range coherence scale. Results are summarized in Sec. VI.

\section{COHERENCE SCALE EXPRESSIONS}

In this section, the geometry of the problem is defined and geometric arguments are used to derive the relationships
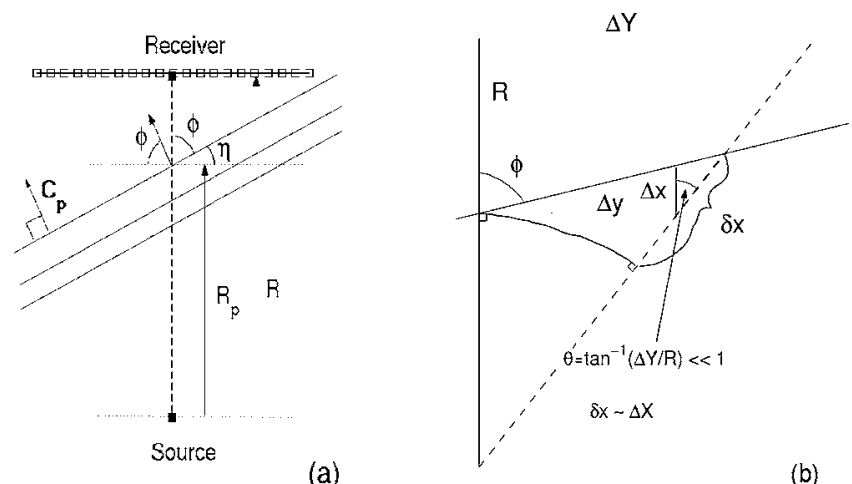

(a)

(b)

FIG. 1. (a) Definition sketch for wave/acoustic angle $\phi$, source-receiver range $R$, source-wave packet range $R_{P}$, and cross-range tangent receiver plane. The angle $\eta$ that is complementary to $\phi$ is also shown. The usual right-handed three-dimensional coordinates are used, with the $x$ direction pointing upward parallel with the arrow indicating $R$, the $y$ direction pointing to the left, and the $z$ direction out of the page. Angle $\phi$ ranges from -90 to 90 , with a positive example shown. The cross-range tangent plane $\mathbf{P}$ used for coherence studies rises out of the page from the receiver line (open squares), with position $y=0$ at the filled square at distance $R$ from the source, (b) The right-hand side of (a) is reproduced. Line segments are defined for sound traveling along the dashed line from the source at the bottom of the triangle to a receiver in the tangent plane at cross-range distance $\Delta Y$ at the upper right. The left-hand edge of the triangle (line segment $R$ ) connects the source with a receiver at $\Delta Y=0$. The line segments $\Delta Y$ in the tangent plane, $\Delta y, \Delta x$, and $\delta x$ are shown, along with angle $\phi$. These line segments have lengths equal to their names, i.e., $R$ has length $R$. An internal wave trough at angle $\phi$ with respect to $R$ is drawn. If $\Delta Y / R$ is sufficiently small, $\delta x$ is approximately equal to $\Delta x$, which is easy to calculate.

between $T_{c}$ and $S$ and between $Y_{c}$ and $S$, respectively. A diagram of source, receiver, and wave packet positions is shown in Fig. 1(a). The $x$ direction is defined to be parallel to the source-receiver path. The source to receiver distance is denoted $R$. The source to packet distance along the sourcereceiver path is denoted $R_{P}$. The angle between the wave packet propagation direction (aligned with the velocity vector $\mathbf{C}_{\mathbf{P}}$ ) and the acoustic path is $\eta$, which has complementary angle $\phi$. Thus, $\phi$ is the angle between the wave crests (or single wave crest, wave trough, or wave troughs) and the acoustic propagation path. The cross-range tangent vertical plane, which is normal to the acoustic path, and within which we will examine cross-range coherence, is denoted $\mathbf{P}$. Horizontal lines within $\mathbf{P}$ are parallel to the unit vector in the $y$ direction.

The temporal coherence scale $T_{c}$ follows directly from knowledge of $S$ for the waveguide and internal wave forms under consideration, and of the characteristics of how those waves propagate. Allowing for the translation of the wave or packet at phase velocity $C_{p x}$ in the direction of acoustic propagation gives $T_{c}=\left|S / C_{p x}\right|$. The phase velocity in the acoustic propagation direction can easily be computed for waves moving in any direction. If the waves are traveling at phase speed $C_{p}$ in the direction $\eta$ relative to the acoustics, as shown in Fig. 1(a), then $C_{p x}=C_{p} / \cos \eta$. Substitution gives $T_{c}=\left|S \cos \eta / C_{p}\right|$. An equivalent expression is

$$
T_{c}=\left|S \sin \phi / C_{p}\right| .
$$

Figure 2 shows a contour plot of $T_{c}$ with $C_{p}=0.8 \mathrm{~m} / \mathrm{s}$. For given $S$ and $C_{p}$, note that the longest time scales are asso- 


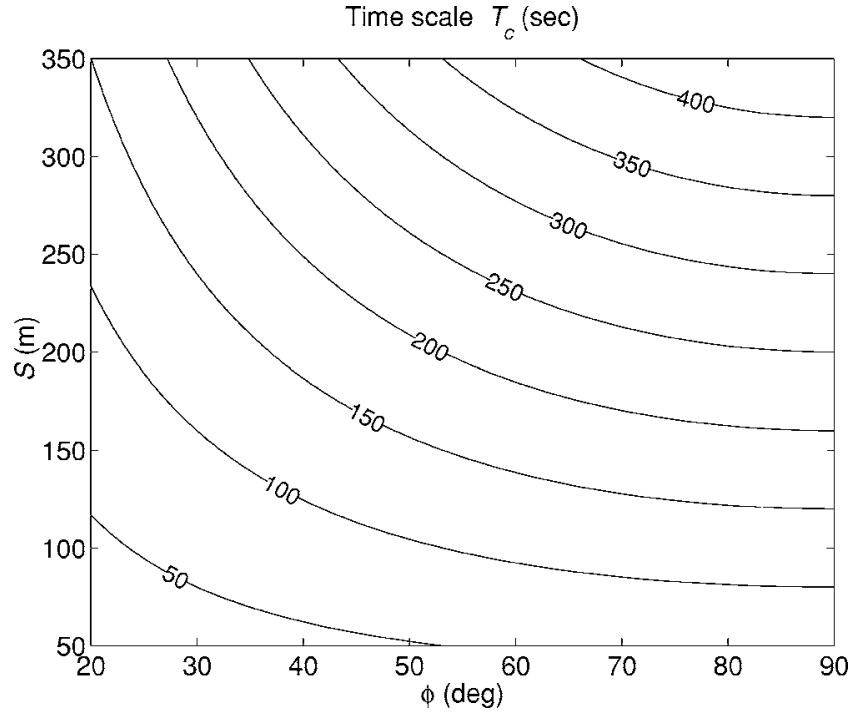

FIG. 2. Time scale $T_{c}(\phi, S)$ is contoured as a function of $S$ in meters and $\phi$ in degrees for $\mathbf{C}_{\mathbf{p}}=0.8 \mathrm{~m} / \mathrm{s}$.

ciated with $\phi$ equal to $90^{\circ}$. This is because $C_{p x} / C_{p}$ has its minimum of unity at that angle. However, the use of a fixed value of $S$ for waves of differing $\phi$ (but having an identical form) must be recognized as an implicit assumption or as an approximation. This is because the coupling process of sound in the $x$ direction can be altered by the effective stretching of the wave scale for $\phi$ not equal to $90^{\circ}$ (Oba and Finette, 2002; Preisig and Duda, 1997). Nonetheless, (1) describes the coherence time as a function of $S$, wave angle, and wave speed, despite the selection of a single $C_{p}$ for the display. An alternative display of $T_{c} / S$ contours with respect to $C_{p}$ and $\phi$ would show other aspects of the behavior.

The $T_{c}$ values given in Fig. 2 compare well with coherence times of $100 \mathrm{~Hz}$ bandwidth pulses measured during the active wave period of the South China Sea component of the Asian Seas International Acoustics Experiment. $T_{c}$ values for two propagation paths, $32 \mathrm{~km}$ upslope and $21 \mathrm{~km}$ alongshelf, have been published (Chiu et al., 2004; Duda et al., 2004). These were computed from correlation functions of depthaveraged intensity using a threshold of 0.707 . Published numbers range from approximately 40 to $220 \mathrm{~s}$. The numbers can be recomputed using a decorrelation threshold of 0.5 in order to be consistent with the remainder of this paper. During the active wave period of 6-13 May 2001, the east (alongshelf) path coherence times, computed for one-day long averaging intervals, were 60 to $100 \mathrm{~s}$, with most values near $70 \mathrm{~s}$. For a wave along this path, good estimates of $C_{p}$ and $\phi$ are $1.4 \mathrm{~m} / \mathrm{s}$ and $55^{\circ}$, respectively. Using these values, $S$ values obtained using (1) range from 100 to $180 \mathrm{~m}$, with most values near $120 \mathrm{~m}$. These measurements are entirely consistent with the assumptions and results of this paper. Additional coherence time estimates from this experiment ranging from 1 to $15 \mathrm{~min}$, computed using energy in a narrow frequency band, appear in Mignerey and Orr (2004).

Next, an expression for the coherence scale $Y_{c}$ along a cross-range line is derived. The expression pertains to the situation of a reception distant from a source such that $S / R$ $\ll 1$, and such that $R$ is greater than the cross-wave (aligned with $\mathbf{C}_{\mathbf{P}}$ ) packet scale length. Adding an assumption that the lateral cross-range separations $\Delta Y$ to be considered adhere to $\Delta Y / R \ll 1$, then $\Delta Y$ on a line tangent to a circle at radius $R$ can be approximated by $R \Delta \psi$, where $\psi$ is azimuth and $\Delta \psi$ is azimuthal separation. (Such a line is contained in plane $\mathbf{P}$.) Because the azimuth along which sound travels is a function of $\Delta Y$, the loss of sound coherence at increasing $\Delta Y$, with respect to sound at $\Delta Y=0$, where the tangent line touches the circle, is caused by sound at differing $\Delta Y$ encountering the packet at differing $R_{P}$. If these differing $R_{P}$ values surpass the value of $S$ for sufficiently high $\Delta Y$, then coherence is small.

$Y_{C}$ depends on the normalized range of the wave packet, $R_{N}=R_{P} / R$, which is between zero and one. This is evident by observing that the lateral separation at range $R_{P}$ of sound encountering packets at azimuthal separation $\Delta \psi$ is $\Delta y$ $=R_{N} \Delta Y=R_{N} R \Delta \psi$, so that near the limit of $R_{N}=0$ all sound reaching plane $\mathbf{P}$ at $\Delta Y<R$ will pass through a very restricted region of the packet.

Now, notice that $\Delta y$ is associated with a perturbation of the packet distance from the source (measured with respect to the packet distance to source for $\Delta Y=0)$ given by $\delta x$ $\sim \Delta x=\Delta y \tan \eta$. An assumption used here is that the angle $\theta=\tan ^{-1}(\Delta Y / R)$ is small, which is fully consistent with the assumption of small cross-range apertures with respect to $R$ [Fig. 1(b)]. Now, to find the cross-range correlation length scale $\Delta Y_{c}$, equate the decoherence displacement scale $S$ to $\delta x$. This converts $\delta x=R_{N} \Delta Y \tan \eta$ into $S=R_{N} Y_{c} \tan \eta$. Thus, $Y_{c}=S\left(R / R_{P}\right) / \tan \eta$, which can be written as

$$
Y_{c}=S\left(R / R_{P}\right) \tan \phi .
$$

For the chosen situation of $\Delta Y / R \ll 1$, the expression can be used to describe either an azimuthal (along an arc of equal distance from the source) or a cross-range (linear) correlation. $Y_{c} / S$ is related in a simple way to $R_{N}$ and $\tan \phi$.

Figure 3 shows the length scale $Y_{c}$ normalized against $S$ and contoured as a function of $R / R_{P}$ and $\phi . Y_{c}$ is one to four times $S$ for roughly half the diagram. It is very large for $\phi$ near $90^{\circ}$, exceeding the final contour indicating $Y_{c}=40 \mathrm{~S}$. This is because the range at which sound encounters the packet, and at which mode coupling commences, is a weak function of sound propagation azimuth. At lower $\phi$, the encounter range is a strong function of azimuth, causing sound to decorrelate at relatively small $\Delta Y$, and giving a shorter correlation scale.

\section{COMPUTATIONAL STUDY OF CROSS-RANGE COHERENCE}

Results of numerical simulations of sound traveling through internal wave packets are presented in this section. These are shown for two purposes: To verify the validity of the cross-range coherence scale expression (2), and to obtain estimates of the scale $S$. These simulations are similar in nature to those appearing in previous publications (Duda, 2004; Duda and Preisig, 1999). The previous simulations were of propagation along single paths, and the papers report quatities such as coherence time scales $T_{c}$, single-point intensity variances, depth-averaged intensity variances, and inten- 


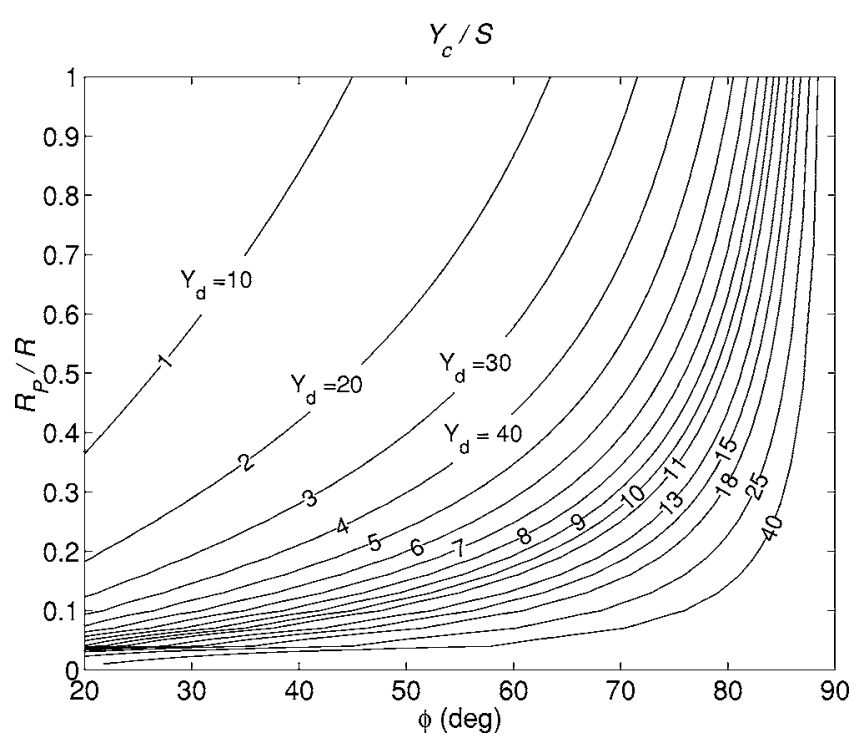

FIG. 3. Cross-range coherence scale $Y_{c}\left(\phi, R_{P} / R\right)$, divided by $S$ for normalization, is contoured. The strictly numerical labels of the contours indicate $Y_{c} / S$. The other labels indicate nondimensional $Y_{d}$, where the representative value $S / \lambda=10$ has been used (see Table III.)

sity biases compared to a wave-free state. Mode-coupling behavior was also diagnosed. To examine cross-range coherence, the new simulations must have an expanded scope, with $N \times 2$-D or 3-D computations taking the place of the single-plane computations. $N \times 2$-D computations are chosen for use here, as explained in the introduction.

The results reported in this section are for monochromatic $200 \mathrm{~Hz}$ sound. A second set of simulations, reported in Sec. IV, are for monochromatic sound at selected frequencies between 100 and $900 \mathrm{~Hz}$. The simulations are of gently upslope propagation from water having $130 \mathrm{~m}$ depth at the source to water having $70 \mathrm{~m}$ depth at the receiver plane $\mathbf{P}$, $27 \mathrm{~km}$ from the source. The bathymetry is shown in Fig. 4. The source is $100 \mathrm{~m}$ below the surface unless specified to be $20 \mathrm{~m}$, as it is in a few cases.

\section{A. Description of the computations}

A version of the RAM code originating at the Naval Research Laboratory is used. The input and output routines, and bottom parameter allocation within the depth grid, have been modified from the original code (RAM 1.1). The code uses methods developed by Collins and collaborators (Collins and Westwood, 1991). The following parameters are used: range step $0.5 \mathrm{~m}$; depth grid interval $0.25 \mathrm{~m}$; Pade expansion parameter 2. Attenuation in the seafloor is $\beta$ $=0.1 \mathrm{db} /$ wavelength to a depth of $6 \mathrm{~m}$, then increases linearly to $\beta=10 \mathrm{db} /$ wavelength at $50 \mathrm{~m}$ into the seabed, so that $\beta=1$ at about $12 \mathrm{~m}$ depth. (This attenuation is unrealistically high and is the result of a coding error. Some simulations were repeated with the intended value of $\beta=0.1$ for the top $50 \mathrm{~m}$ of the seabed, and the results were the same. The results are also relatively insensitive to the seafloor sound speed and seafloor density.)

The $N \times 2$-D simulations of propagation through moving 3-D wave packet structures are accomplished by organizing $N$ runs at a varying azimuth through wave structures, as (a)

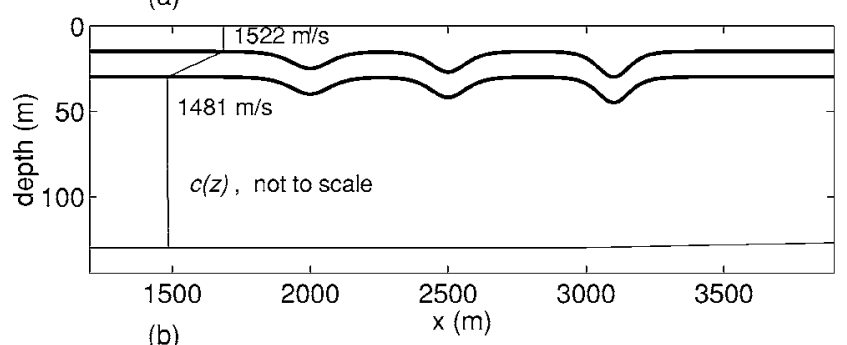

(b)

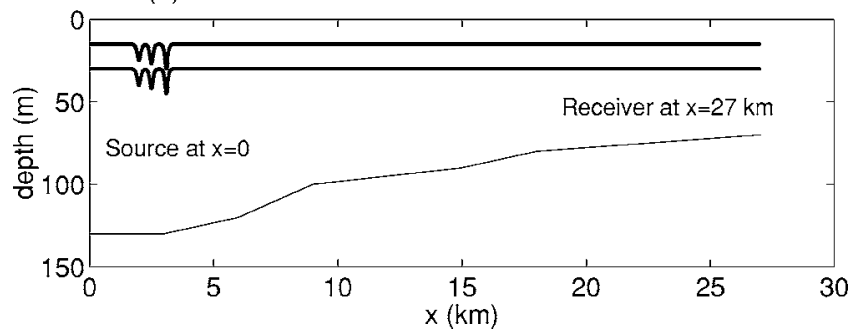

FIG. 4. (a) The background sound speed profile and the three-wave packet geometry used in the simulations are shown. The thick lines show the interfaces between the isovelocity upper layer, the gradient layer and the nearisovelocity lower layer. The waves are shown at packet position $R_{P}$ $=2000 \mathrm{~m}$. The wave shapes are shown in a plane normal to the wave troughs, which is parallel to their direction of propagation. The distances between the wave crests are 500 and $600 \mathrm{~m}$. The waves have amplitudes $a$ of 10,12 , and $15 \mathrm{~m}$, and horizontal scales $L$ of 95,86 , and $77 \mathrm{~m}$, agreeing with solutions to the $\mathrm{KdV}$ equation for a density profile that is consistent with the sound speed profile, (b) The water depth between the source in $130 \mathrm{~m}$ deep water and the receiver plane in $70 \mathrm{~m}$ deep water is shown. The waves seen in panel (a) are shown again.

shown in the plan view in Fig. 1, for each packet position $R_{P}$. Thus, an investigation of $M$ values of $R_{P}$ would require $N$ $\times M$ runs. The runs terminate at a tangent plane at $R$ $=27 \mathrm{~km}$, with $\Delta Y$ ranging from 0 to $1039 \mathrm{~m}$, with associated azimuthal propagation ranges of 27 to $27.02 \mathrm{~km}$. The azimuths are chosen such that the termination ranges are integer multiples of the range step of $0.5 \mathrm{~m}$, resulting in an unequal $\Delta Y$ sampling that causes no difficulty. The $27 \mathrm{~km}$ run (the shortest) has a packet at $R_{P}$, with packet positions for the other runs (i.e., those with $\Delta Y \neq 0$ ) computed using analytic geometry.

The speed and density profiles used in the simulations are similar to those used previously (Duda, 2004), with the exception that a higher subseafloor sound speed is used here. The sound-speed profile in the seabed is not a function of range. At the, seafloor the speed is always $1600 \mathrm{~m} / \mathrm{s}$ regardless of the water depth, and has a gradient of $1 \mathrm{~m} / \mathrm{s}$ for the top $50 \mathrm{~m}$ of the seafloor. At $50 \mathrm{~m}$ depth in the seabed, it then rises from 1650 to $2400 \mathrm{~m} / \mathrm{s}$ to simulate the underlying bedrock.

Packets are composed of three waves, each of form $\zeta(x, t)=a \operatorname{sech}^{2}[(x-c t) / L]$, which are solutions of the Korteweg-de Vries (KdV) equation (Lamb and Yan, 1996; the Lee and Beardsley, 1974). The wave shapes with respect to range must be stretched by $1 / \cos \eta$ within each of the propagation planes. An additional correction of stretching as a function of the azimuth, required for the wave shapes to be strictly correct, is not needed because the azimuthal domain is small.

Unlike earlier 2D simulations (Duda and Preisig, 1999; Preisig and Duda, 1997), these 3-D simulations require that $a$ 

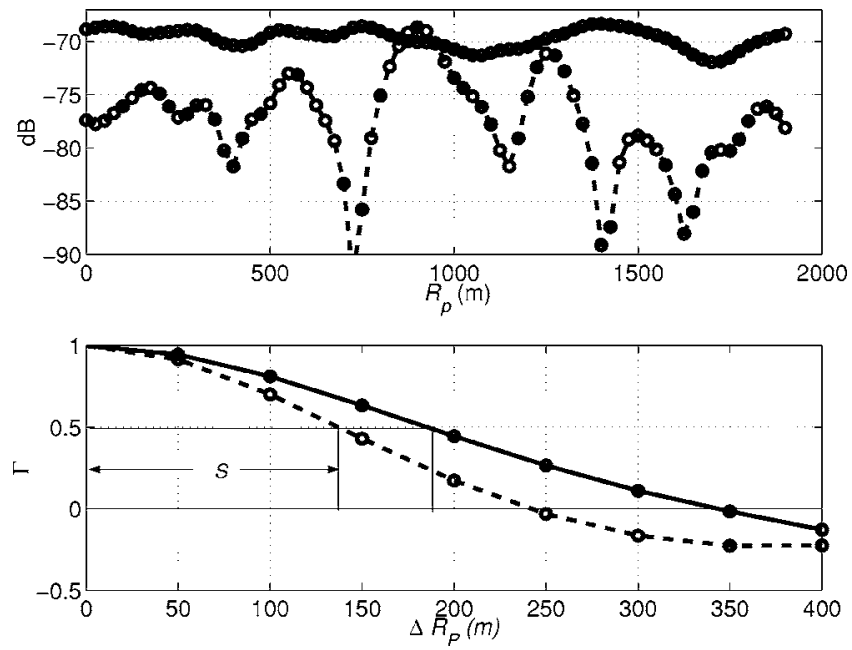

FIG. 5. (Lower panel) Correlation functions are shown for near bottom (dashed line) and depth-averaged (solid line) intensity at a $27 \mathrm{~km}$ receiver for $\phi=70^{\circ}$ moving-packet $200 \mathrm{~Hz}$ simulations. The calculation is made for wave packets at ranges $R_{P}=0$ to $1900 \mathrm{~m}$ from the source, with a $25 \mathrm{~m}$ increment. Decorrelation displacement scales $S$ estimated from the correlation functions are indicated. (Upper panel) The intensity fields from which the correlation functions are computed are shown. Two are shown: The depth-averaged water-column intensity (solid line) and the intensity at the bottom (dashed line). The scale is signal arriving from a $0 \mathrm{~dB}$ source (i.e., the additive inverse of transmission loss.)

and $L$ be chosen for each wave such that $\zeta$ are solutions of $\mathrm{KdV}$ (i.e., properly scaled waves must be used, then $1 / \cos \eta$ stretching applied, rather than using a variety of $L$ for a given $a$ to represent a variety of $\eta$ ). Appropriate wave parameters for a density profile consistent with our sound-speed profile are $L: a$ pairs of 95:10,86:12, and 77:15, with all dimensions in meters. Phase speeds for these waves are near $\left|\mathbf{C}_{\mathbf{P}}\right|$ $=0.73 \mathrm{~m} / \mathrm{s}$. Figure 4 shows water column (sound-speed profile) displacements in the acoustic propagation plane for an example wave packet at $\eta=0^{\circ}\left(\phi=90^{\circ}\right)$, so that the wave shapes are not stretched, as they would be for other values of $\eta$. The waves do not evolve over time in our simulations, unlike their true behavior. This means that acoustic coherence over long time scales cannot be addressed. This is not an important concern because the results shown here are consistent with the rapid loss of coherence, and adding wave packet evolution (time scale tens of minutes) would reduce coherence scales below the already small values shown here.

\section{B. Results}

First, estimates of $S$ from the $200 \mathrm{~Hz}$ simulations will be examined, then estimates of $Y_{c}$ will be presented. Figure 5 shows the depth-averaged intensity and near-bottom intensity at a single point $(\Delta Y=0)$ as a function of $R_{P}$ for a $\phi=70^{\circ}$ simulation with the packet near the source. The crosscorrelation function given by $\Gamma\left(\Delta R_{P}\right)=\left\langle F\left(R_{P}\right) F\left(R_{P}+\Delta R_{P}\right)\right\rangle$ is shown, with $\Delta R_{P}$ normalized by the acoustic wavelength. The angle brackets indicate ensemble average, computed in this paper by averaging results over small ranges of $R_{P}$. The variable $F$ is either the depth-averaged or near-bottom intensity with the mean removed, normalized such that $\Gamma(0)=1$. The point where $\Gamma$ drops to 0.5 is used here as the decorre-
TABLE I. Computed $Y_{c}$ and $S$ values from $200 \mathrm{~Hz}$ simulations are given for various wave angles $\phi$ and scaled packet ranges $R_{N}=R_{P} / R . Y_{c}$ is reported in ratio form, $Y_{c} / S$. The theoretical ratio $Y_{c} / S$ is also reported. Both $S$ and $Y_{c}$ are computed using the near-bottom field (for case 1, the dashed lines in Fig. 5). The notation nc means not computed. The value with the asterisk is extrapolated from a correlation function curve that has a value near 0.6 at the longest computed lag, above the threshold of 0.5 .

\begin{tabular}{lccccc}
\hline \hline Identifier & $\phi\left(^{\circ}\right)$ & $R_{N}$ & $S(\mathrm{~m})$ & $Y_{c} / S$ simulated & $Y_{c} / S$ using (2) \\
\hline Case 1 & 70 & 0.04 & 140 & nc & 69 \\
Case 2 & 70 & 0.18 & 118 & $10^{*}$ & 15 \\
Case 3 & 70 & 0.50 & 77 & 5.7 & 5.5 \\
Case 4 & 70 & 0.93 & 73 & 3.7 & 3.0 \\
Case 5 & 50 & 0.50 & 78 & 2.5 & 2.4 \\
Case 6 & 50 & 0.93 & 79 & 1.4 & 1.3 \\
\hline \hline
\end{tabular}

lation scale. The correlation drops to one-half at the indicated $S$ values of 190 and $140 \mathrm{~m}$ for the two measures of intensity, respectively.

Table I gives $S$ values for the simulation shown in Fig. 5 (case 1), and other cases numbered 2 through 6 covering over a variety of $\phi$ and $R_{N}$. In each case the correlation function used to compute the scale stems from an ensemble average taken over a small range of $R_{N}$. Therefore the $R_{N}$ values in Table I are merely representative for each case. It would be preferable for $S$ to be uniform over $\phi$ and $R_{N}$, which it is not, because then (1) and (2) would be most useful. $S$ is reasonably uniform, however, except at small $R_{N}$.

To illustrate the lateral decorrelation effect parameterized by $Y_{c}$, representative images of computed acoustic fields in the cross-range tangent plane $\mathbf{P}$ are now shown. Figure 6 shows a snapshot of the intensity in this plane for a near-
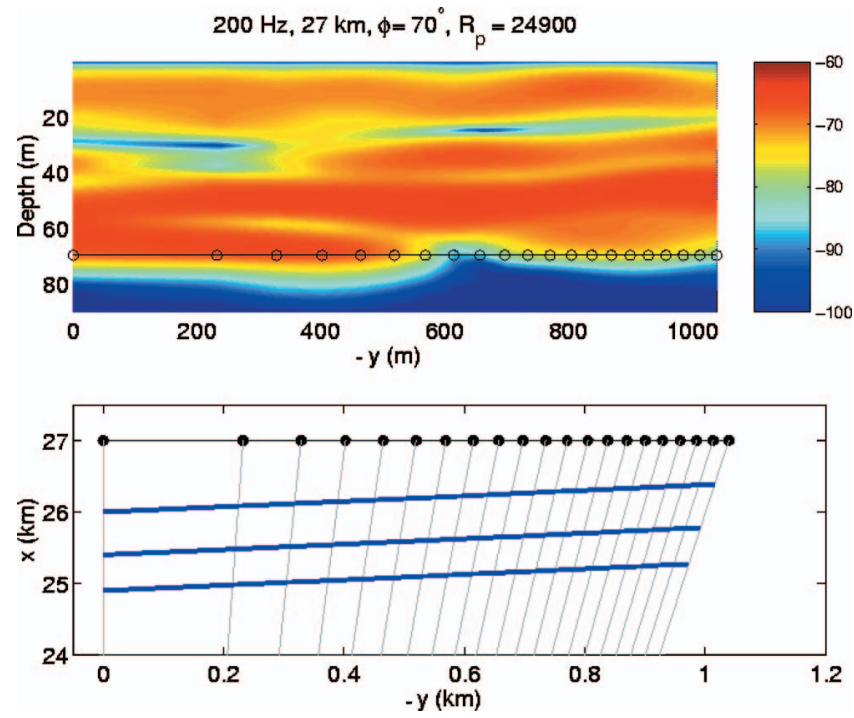

FIG. 6. (Upper panel) The intensity in the tangent plane $\mathbf{P}$ is contoured. The signal level in $\mathrm{dB}$ from a $0 \mathrm{~dB}$ source is shown. The left edge is $27 \mathrm{~km}$ from the source $(200 \mathrm{~Hz}$, at the standard $100 \mathrm{~m}$ depth); the right edge is $27.02 \mathrm{~km}$ from the source. Results with packet angle $\phi=70^{\circ}$ at $R_{P}=24.9 \mathrm{~km}$ are shown. The line of circles show the positions that bottom mounted receivers at the terminations of the $N$ azimuthal propagation lines would have. (Lower panel) The positions of the wave troughs in the three-wave packet corresponding to the upper panel results are shown in this plan view. The acoustic source is at $(0,0)$. The azimuthal propagation lines are shown. The intersection of the receiver plane $\mathbf{P}$ with the seafloor is shown with the black symbols. 

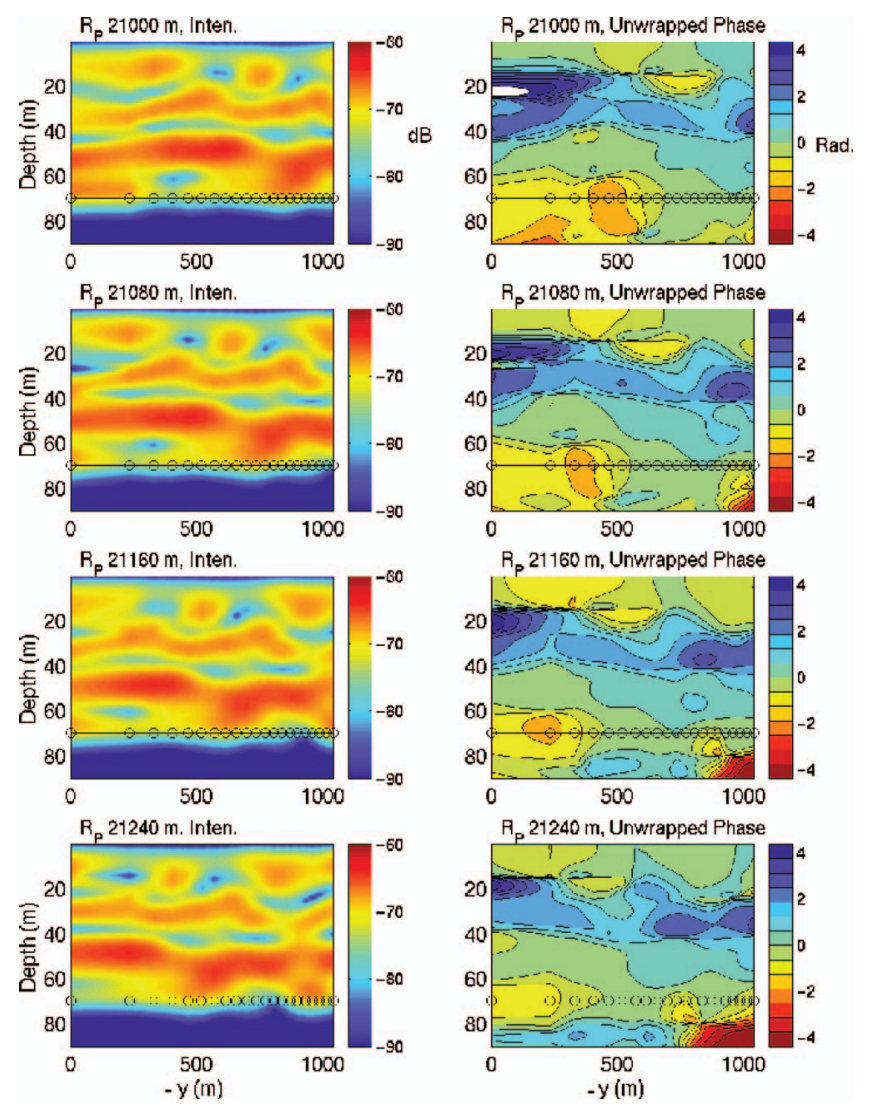

FIG. 7. Intensity and phase in the cross-range (tangent) plane $\mathbf{P}$ at range $27 \mathrm{~km}$ are shown for a $\phi=50^{\circ}$ packet at four different $R_{P}$. The acoustic frequency is $200 \mathrm{~Hz}$ and the source depth is $20 \mathrm{~m}$. The format is similar to the upper panel of the previous figure. The $R_{P}$ are 21000 to 21240 in $80 \mathrm{~m}$ intervals, shown from top to bottom. For each of the four $R_{P}$, the intensity is contoured with a continuous color map at the left, and the unwrapped phase is contoured in a stepwise fashion at the right. The same section of $\mathbf{P}$ is shown in each panel. The viewpoint is such that sound would be propagating into the page. The fluctuation features that can be seen are typical for simulations over a range of frequencies, $R_{P}$, and $\phi$. It is evident that the features move from right to left in the figure as the packet moves toward the receiver.

receiver wave packet. The figure is drawn such that sound would be propagating into the page. This snapshot is from case 4 (Table I). The intensity exhibits features with scale length $Y_{c}$ of approximately $270 \mathrm{~m}$. Next, Fig. 7 shows four sequential snapshots of fluctuating cross-range fields from simulated moving packets. The $R_{P}$ increment is $80 \mathrm{~m}$, and $R_{P}$ is an increasing function of time, so that time would progress down the page. Intensity and phase are contoured on the left and right, respectively. Features are seen to move from right to left with increasing $R_{P}$ or time. This is because the range of the packet from the source is a decreasing function of azimuth from right to left, as illustrated in Fig. 1. In this situation, acoustic field features, which are closely linked to the packet range from the source (Duda and Preisig, 1999), move from right to left as time progresses. Finally, note that cross-range features have a smaller scale length for this $\phi=50^{\circ}$ case than for the $\phi=70^{\circ}$ case of Fig. 6 .

Table I includes $Y_{c} / S$ computed directly from simulations for cases 3-6. These are computed from lagged correlation functions of the complex field, $C(\Delta Y)=\left\langle\Psi(\Delta Y) \Psi^{*}(Y\right.$ $=\Delta Y)\rangle$. (For case $1, C(\Delta Y)=0.96$ at the longest lag, so the
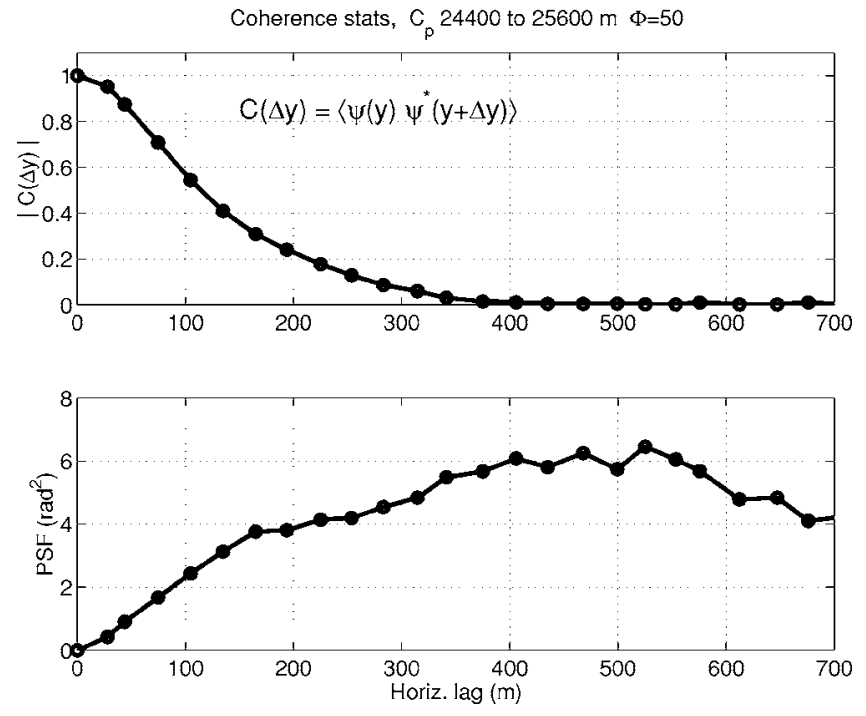

FIG. 8. (Upper panel) The magnitude of the complex cross-range lagged correlation function for a near-bottom $200 \mathrm{~Hz}$ acoustic field is shown for the case $\phi=50^{\circ}, 24.4<R_{P}<25.6 \mathrm{~km}$. The source depth is the standard $100 \mathrm{~m}$. This is computed after normalizing the amplitudes across the array so that they average unity at each position, to account for the slightly different range to each position. This ensures that the value at zero lag is one. (Lower panel) Mean squared phase difference for the same data. The points shown in each panel are averages taken over $R_{P}$ and over a few different lags falling into lag bins. The points shown are (average lag, average product) for each lag bin.

coherence scale is very long and was not computed; for case 2 with the asterisk, $C$ was extrapolated beyond the maximum available lag of $\Delta Y=950 \mathrm{~m}$.) All of these simulation-based $Y_{c}$ values are derived from near-bottom cross-range acoustic field properties, as explained in the next paragraph. Except for one value, these agree well with the values of $Y_{c} / S$ computed from only $R, R_{P}$, and $\phi$ using (2), which are also listed in the table. The value that does not agree well is for case 2 , which is a situation having waves very close to the source. In this situation, the computed correlation length is shorter than that given by the simple expression. A probable reason for this is that the curved line of constant range from source seen in Fig. 1(b) departs from the dashed line of length $\Delta y$, so that the approximation $\delta x=\Delta x$ breaks down, with $\delta x>\Delta x$.

Figure 8 shows a typical lagged correlation function of the complex field, $C$, from which the computational $Y_{c}$ estimate is derived. Case 6 results are shown. The complex fields are tabulated as functions of cross-range horizontal lag and $R_{P}$, then normalized so that they have an average magnitude of one at each cross-range position. The crossproducts are then computed, and finally the results are ensemble averaged to obtain meaningful averages at each lag. This process eliminates the (very small) effect of a weak intensity trend generated by the differing ranges to the various $\Delta Y$ points. The lower panel of Fig. 8 shows the phase structure function (the mean squared phase difference $\left.\operatorname{PSF}(\Delta y)=\left\langle[\varphi(y)-\varphi(y+\Delta y)]^{2}\right\rangle\right)$ computed from the same dataset. The rapid increase of phase difference as a function of lag indicates that phase changes are responsible for much of the cross-range decorrelation within the complex field. 

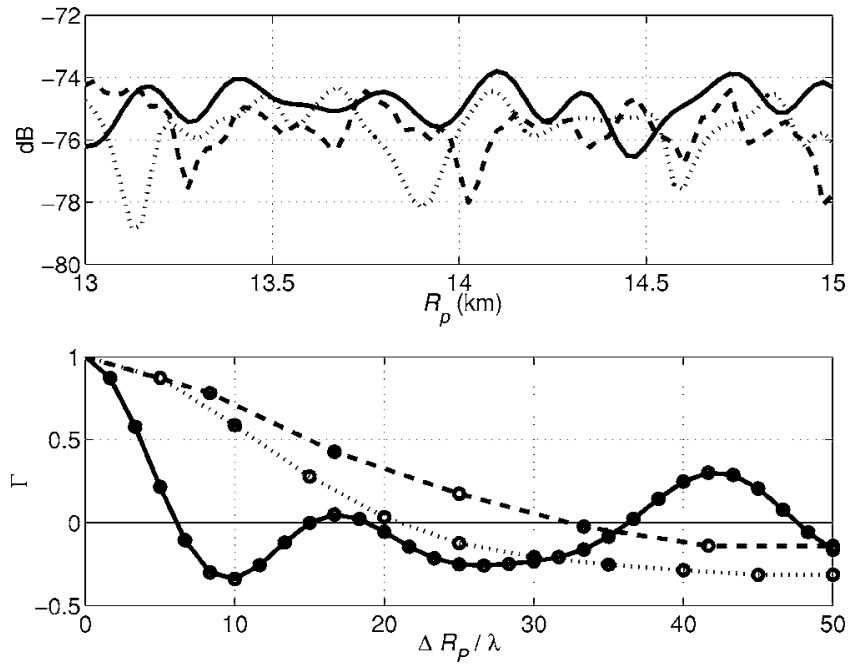

FIG. 9. (Upper panel) For moving wavepacket propagation runs having $\phi$ $=70^{\circ}$ and source depth $20 \mathrm{~m}$, depth-integrated intensity $E$ within the water column is shown as a function of $R_{P}$ for three frequencies: $100 \mathrm{~Hz}$ (solid); $300 \mathrm{~Hz}$ (dot); and $500 \mathrm{~Hz}$ (dash). (Lower panel) The cross-correlation function $\Gamma=\left\langle E_{f}\left(R_{P}\right) E_{f}\left(R_{P}+\Delta R_{P}\right)\right\rangle /\left\langle E_{f}^{2}\right\rangle$ is shown, with $\Delta R_{P}$ normalized by the acoustic wavelength. $E_{F}$ is $E$ with the mean removed and normalized such that $\Gamma(0)=1$.

\section{COMPUTATION OF S FOR A RANGE OF FREQUENCIES}

The presence of the displacement decorrelation scale $S$ in (1) and (2) means that it plays a critical role in determining the scales of temporal and cross-range coherence $\left(T_{c}\right.$ and $Y_{c}$ ), at least for the idealized cases of nonevolving and slowly evolving wave packets. This role was validated for $200 \mathrm{~Hz}$ sound in the previous section (Table I). Therefore, uncovering the frequency $(f)$ dependence of $S$ will help divulge the frequency dependence of $T_{c}$ and $Y_{c}$. Here, this issue is given an initial exploratory treatment: Our simulations are extended to other frequencies and $S(f)$ is computed for $100<f<900 \mathrm{~Hz}$. In general, as pointed out previously, mode coupling in internal waves depends on many aspects of acoustic waveguide vertical structure, wave amplitudes, and horizontal scales (Duda and Preisig, 1999; Preisig and Duda, 1997), so analysis of the frequency dependence of the problem is beyond the scope of this paper.

Correlation functions $\Gamma\left(\Delta R_{P}\right)$ of depth-integrated intensity for moving packets at three frequencies $(100,300$, and $500 \mathrm{~Hz}$ ) are shown in the lower panel of Fig. 9. These can be compared with Fig. 5, although the packet-location lags in Fig. 9 are normalized by an acoustic wavelength. The series from which the correlation functions are computed are shown in the upper panel. The same geometry is used as in all previous simulations. The figure shows $S$ to range from 4 to 15 times the acoustic wavelength. The intensity fluctuation variances, not tabulated here, are comparable to those resulting from previous simulations (Duda, 2004; Duda and Preisig, 1999).

Estimates of $S$ derived from $\Gamma$ curves such as those of Fig. 9 were obtained for frequencies from 100 to $900 \mathrm{~Hz}$, and are given in Tables II and III. Table II shows values for the case of a $100 \mathrm{~m}$ source depth. Table III shows values for the case of a $20 \mathrm{~m}$ source depth. These estimates of $S$ are
TABLE II. $S$ is computed for various frequencies. The wave angle $\phi$ is $70^{\circ}$ and the $R_{P}$ range is from 13 to $15 \mathrm{~km}$, so that $R_{P} / R \approx 0.5 . S$ is computed for near-bottom intensity and for depth-integrated intensity. The source depth is $100 \mathrm{~m}$. $\lambda$ is the acoustic wavelength.

\begin{tabular}{lcccc}
\hline \hline $\begin{array}{l}\text { Frequency } \\
(\mathrm{Hz})\end{array}$ & $\begin{array}{c}S / \lambda \\
\text { nr. btm. }\end{array}$ & $\begin{array}{c}S / \lambda \\
\text { depth int. }\end{array}$ & $\begin{array}{c}S \\
\text { nr. btm. }(\mathrm{m})\end{array}$ & $\begin{array}{c}S \\
\text { depth int. (m) }\end{array}$ \\
\hline 100 & 6 & 4 & 87 & 66 \\
200 & 11 & 9 & 85 & 66 \\
300 & 7 & 8 & 36 & 42 \\
400 & 11 & 14 & 40 & 52 \\
500 & 10 & 16 & 31 & 49 \\
600 & 11 & 13 & 27 & 33 \\
700 & 10 & 18 & 21 & 39 \\
800 & 12 & 13 & 23 & 24 \\
900 & 11 & 23 & 19 & 39 \\
\hline \hline
\end{tabular}

given in dimensional units of length, and also in nondimensional form after normalization using the acoustic wavelength $\lambda$. The results shown in Fig. 9 are included in Table III. The $200 \mathrm{~Hz}$ near-bottom $S$ value of $85 \mathrm{~m}$ in Table II is from a run similar but not identical to the run used to compute the directly comparable $200 \mathrm{~Hz} S$ value of $77 \mathrm{~m}$ in Table I, with the runs having differing $R_{P}$ spacing and window size. These differing $S$ demonstrate the practical confidence intervals on $S\left(\phi, R_{N}, f\right)$.

The values of $S\left(70^{\circ}, 0.5, f\right)$ for a $100 \mathrm{~m}$ source depth for the chosen waveguide and source-receiver parameters, given in Table II, are not strictly constant with respect to frequency in either dimensional or nondimensional form. The quantity closest to being constant is the nondimensional form $S / \lambda$ computed for the near-bottom field, with a value of about $S / \lambda=10$. The corresponding $S$ decrease strongly with increasing frequency. On the other hand, when computed from the depth-integrated intensity, the dimensional $S$ has a more constant value with respect to frequency than the nondimensional $S / \lambda$. However, $S$ derived from the depthintegrated intensity decreases by approximately a factor of 2 , as frequency increases from 100 to $900 \mathrm{~Hz}$.

The same two statements can be made for the $20 \mathrm{~m}$ source depth case (Table III), namely, that the nondimensional near-bottom $S / \lambda \approx 10$, and that, for the depthintegrated field, dimensional $S$ is a weaker function of fre-

TABLE III. $S$ is computed for various frequencies. All parameters of the runs are identical to those reported in Table II, with the exception that the source depth is $20 \mathrm{~m}$. The fluctuations of the near-bottom series are 4 to $6 \mathrm{~dB}$ rms. Fluctuations of the depth integrated series are near $1.0 \mathrm{~dB}$ rms.

\begin{tabular}{lcccc}
\hline \hline $\begin{array}{l}\text { Frequency } \\
(\mathrm{Hz})\end{array}$ & $\begin{array}{c}S / \lambda \\
\text { nr. btm }\end{array}$ & $\begin{array}{c}S / \lambda \\
\text { depth int. }\end{array}$ & $\begin{array}{c}S \\
\text { nr. btm }(\mathrm{m})\end{array}$ & $\begin{array}{c}S \\
\text { depth int. }(\mathrm{m})\end{array}$ \\
\hline 100 & 5 & 4 & 73 & 55 \\
200 & 16 & 8 & 117 & 59 \\
300 & 9 & 11 & 43 & 57 \\
400 & 9 & 11 & 34 & 40 \\
500 & 8 & 15 & 24 & 45 \\
600 & 7 & 17 & 18 & 43 \\
700 & 11 & 19 & 23 & 40 \\
800 & 9 & 27 & 17 & 50 \\
900 & 13 & 31 & 22 & 51 \\
\hline \hline
\end{tabular}


quency than nondimensional $S / \lambda$. The first would be even more true in the absence of one outlier of near-bottom $S / \lambda$ (the $200 \mathrm{~Hz}$ entry, which is 16).

The result that is most clear is that $S / \lambda$ ranges from 4 to 31 in this frequency band. Variations within this range depend on how $S$ is computed and on frequency in ways that are yet to be determined. If all the numbers in the two tables are used, nondimensional $S / \lambda$ has a mean of about 12 , with a standard deviation of 6 .

\section{NONDIMENSIONAL CROSS-RANGE COHERENCE SCALE}

A nondimensional cross-range correlation scale can be obtained using the relationship between $Y_{c}$ and $S$ and using $S / \lambda$ results from the preceding section. The scale can be nondimensionalized as follows: $Y_{d}=Y_{c} / \lambda=\left(Y_{c} / S\right)(S / \lambda)$. Figure 3 shows a contour plot of $Y_{c} / S$, and Tables II and III provide $S / \lambda$ values.

For the $100 \mathrm{~m}$ source depth situation (Table II), the value of $S / \lambda=10$ for the near-bottom intensity case allows a numerical conversion of $Y_{c} / S$ into $Y_{d}$, with resultant $Y_{d}$ values indicated in Fig. 3. The contours indicate that approximately half the possible situations show $Y_{d}<50 \lambda$. These are at the upper left. The shortest scales are for waves that are near the receiver having low angle $\phi$ (but not less than $20^{\circ}$, where the assumptions and the simulations both break down).

The situations with longer $Y_{d}$, which are those having high $\phi$, having packets near the source, or having both, are equally likely to exist in nature. This means that field estimates of $Y_{d}$ may take on many values ranging from a few wavelengths to hundreds of wavelengths, depending on the internal wave geometry, interval between packets, whether packets come from one direction or multiple directions, and so on.

The location of sources and receivers with respect to generation sites of nonlinear internal wave packets, in particular, may influence which part of Fig. 3 gives the relevant estimate of $Y_{c} / S$ for a particular experiment, and thus the relevant $Y_{d}$. The location may also influence the acoustic propagation and mode coupling, such that $S / \lambda$ may diverge from a constant, or such that it takes on a different value than 10 used in the figure. It is also possible that wave packets traveling in different directions might pass periodically between the source and the receiver. In this case, if each of the classes of waves (assign waves in each direction to a class) caused fluctuations of approximately equal variance, then the overall field-observed correlation scales would be determined by the waves with the shortest scales $Y_{c}$ and $T_{c}$. Figure 3 suggests that these would be the waves of low $\phi$ close to the receiver, giving correlation lengths of $20 \lambda$ or less.

For a given coastal area, it may be possible to reliably model $S / \lambda$ at the frequency of interest. Subsequently, the source location, receiver location, and local waveguide geometry (obtained via in situ or remote techniques) can then be used to determine $\phi$ and $R_{N}$, yielding $Y_{c} / S$ and $Y_{d}$.

\section{SUMMARY}

We have used two simple models to relate fluctuation effects caused by the presence of internal waves or wave packets to temporal and spatial acoustic coherence scales. The first uses the internal wave velocity to convert the effect of internal wave displacement into a temporal coherence scale. The second uses the plan form geometry of the internal waves to convert the effects of variable internal wave distance from the source into a cross-range coherence scale. Computational simulations give results that agree with predictions from the simple models.

A common factor in the two coherence scale expressions is the internal wave horizontal displacement scale $S$ associated with significant acoustic fluctuation. This scale is governed in part by mode shapes and wave numbers, and depends on details of the environment such as pyncocline geometry, water depth, source depth, and proximity of the waves to either the source or the receiver. Computational examples of how internal wave propagation (horizontal displacement, in the terminology used here) causes acoustic fluctuations can be found here and in other articles (Duda, 2004; Duda and Preisig, 1999; Rouseff et al., 2002).

One of those papers (Duda and Preisig, 1999) contains an explanation of how input mode interference can control the scale $S$. Briefly, the time series of relative amplitudes of various modes on the receiver side of moving packets, which are tied to field fluctuations at the receiver in the absence of further significant coupling, are governed by the spatial beat patterns between the incident modes that couple significant energy into each of the modes. The dimensions of these patterns depend on the mode coupling coefficients, which in turn depend on the depth integral of the product of the coupled mode shapes. Potentially useful expressions governing fluctuations for any waveguide and frequency were included in the paper, but the coefficients and functions in the expressions (mode amplitudes and phases, mode shapes) are difficult to predict because of water column and bottom property uncertainty and variability.

Temporal coherence scales are estimated to be 30 to $120 \mathrm{~s}$, based on $S$ values of 40 to $100 \mathrm{~m}$ (Fig. 2). These correspond well to field estimates at $400 \mathrm{~Hz}$ (Duda et al. 2004) and at 300 and $500 \mathrm{~Hz}$ (Mignerey and Orr, 2004) Spatial scales are estimated to be from a few times the acoustic wavelength up to extremely long values, depending on the position of the waves in the source-receiver path and the angle of the waves with respect to that path.

The temporal coherence scale expression (1) contains the internal wave velocity $\mathbf{C}_{\mathbf{P}}$. This can be obtained from satellite or aircraft remote sensing, pairs of moorings, ship observations, or combinations of these. Thus, the unknown in (1) for a particular site in the field will often be the important scale length $S$. Likewise, the unknown in the coherence scale expression (2) will also often be $S$. As indicated in Sec. IV, $S$ often has values of order $10 \lambda$ (order $50 \mathrm{~m}$ for chosen frequencies), but exhibits a broad range from $4 \lambda$ to $31 \lambda(17-120 \mathrm{~m})$, with greater variation not ruled out. There- 
fore, understanding the behavior of $S$ is critical to the application of the results shown here to prediction of acoustic coherence in the field.

An important consideration is the fact that $S$ may be a function of $\phi$ for a given wave packet. This is not allowed for here, so that the coherence scale formula (2) and Fig. 3 are in fact simplifications that do not reflect that possibility. They must therefore be applied carefully.

The results shown here are for monochromatic acoustic propagation. The problem of pulse spatial variability is more complicated. In a best-case scenario, the correlation behavior would be independent of frequency throughout the entire band of a broadband source (say, with a bandwidth/center frequency ratio of 1/4), and a pulse composed of a sum of frequency constituents might be expected to have behavior similar to that shown here. Alternatively, the changes in mode coupling (and the resultant field after propagation through a packet) that occur as a packet is displaced may be sufficiently frequency dependent for the results shown here to not apply directly to pulse coherence. Analyses of how the acoustic bandwidth affects coherence for signals expressible in terms of sums of Gaussian wave packets (multipath) are contained in Colosi and Baggeroer (2004) and Colosi et al. (2005) and may be useful to apply, or broadband simulations may be used.

One point about the numerical simulations must be noted. In this paper, characteristic values of $S$ were computed from intensity fluctuations rather than from the complex field. This was done in order to be consistent with prior work. On the other hand, $Y_{c}$ values were computed using the complex field correlation function. The complex field was chosen for this because of the role of the phase in array processing and signal processing applications. It is possible that $Y_{c}$ values obtained using the complex field may in some cases be dominated by phase fluctuations, which are not considered in the $S$ evaluations. This means that in some cases the comparison of $S$ with $Y_{c}$ may not precisely fit (2). This only affects the evaluation of (2) via simulation, and does not influence the applicability of (2).

Finally, modifications to the derivation can extend the work to nonbroadside incidence of the signals at the receiver. Such modifications would yield a generalized version of (2).

\section{ACKNOWLEDGMENTS}

The 2-D phase unwrapping code was provided by J. Colosi. This work was funded by grants from the Ocean Acoustics Program of the U.S. Office of Naval Research.
Badiey, M., Katsnelson, B. G., Lynch, J. F., Pereselkov, S., and Siegmann, W. L. (2005). "Measurement and modeling of three-dimensional sound intensity variations due to shallow-water internal waves," J. Acoust. Soc. Am. 117, 613-625.

Chiu, C.-S., Ramp, S. R., Miller, C. W., Lynch, J. F., Duda, T. F., and Tang, T. Y. (2004). "Acoustic intensity fluctuations induced by South China Sea internal tides and solitons," IEEE J. Ocean. Eng. 29, 1249-1263.

Collins, M. D., and Westwood, E. K., (1991). "A higher-order energyconserving parabolic equation for range-dependent ocean depth, sound speed, and density," J. Acoust. Soc. Am. 89, 1068-1075.

Colosi, J. A., and Baggeroer, A. B. (2004). "On the kinematics of broadband multipath scintillation and the approach to scintillation," J. Acoust. Soc. Am. 116, 3515-3522.

Colosi, J. A., Baggeroer, A. B., Cornuelle, B. D., Dzieciuch, M. A., Munk, W. H., Worcester, P. F., Dushaw, D. B., Howe, B. M., Mercer, J. A., Spindel, R. C., Birdsall, T. G., Metzger, K., and Forbes, A. M. G. (2005). "Analysis of multipath acoustic field variability and coherence in the finale of broadband basin-scale transmissions in the North Pacific Ocean," J. Acoust. Soc. Am. 117, 1538-1564.

Duda, T. F. (2004). "Acoustic mode coupling by nonlinear internal wave packets in a shelfbreak front area," IEEE J. Ocean. Eng. 29, 118-125.

Duda, T. F., and Preisig, J. C. (1999). "A modeling study of acoustic propagation through moving shallow-water solitary wave packets," IEEE J. Ocean. Eng. 24, 16-32.

Duda, T. F., Lynch, J. F., Newhall, A. E., Wu, L., and Chiu, C.-S. (2004). "Fluctuation of $400 \mathrm{~Hz}$ sound intensity in the 2001 ASIAEX South China Sea experiment," IEEE J. Ocean. Eng. 29, 1264-1279.

Frank, S. D., Badiey, M., Lynch, J. F., and Siegmann, W. L. (2004). "Analysis and modeling of broadband airgun data influenced by nonlinear internal waves," J. Acoust. Soc. Am. 116, 3404-3422.

Lamb, K. G., and Yan, L. (1996). "The evolution of internal wave undular bores: Comparisons of a fully nonlinear numerical model with weakly nonlinear theory," J. Phys. Oceanogr. 26, 2712-2734.

Lee, C., and Beardsley, R. C. (1974). "The generation of long nonlinear internal waves in a weakly stratified shear flow," J. Geophys. Res. 79, 453-462.

Lynch, J. F., Newhall, A. E., Sperry, B., Gawarkiewicz, G., Fredricks, A., Tyack, P., Chiu, C.-S., and Abbot, P. (2003). "Spatial and temporal variations in acoustic propagation characteristics at the New England Shelfbreak Front," IEEE J. Ocean. Eng. 28, 129-150.

Mignerey, P. C. and Orr, M. H. (2004). "Observations of matched-field autocorrelation time in the South China Sea," IEEE J. Ocean. Eng. 29, 1280-1291.

Oba, R., and Finette, S. (2002). "Acoustic propagation through anisotropic internal wave fields Transmission loss, cross-range coherence, and horizontal refraction," J. Acoust. Soc. Am. 111, 769-784.

Preisig, J. C., and Duda, T. F. (1997). "Coupled acoustic mode propagation through continental-shelf internal solitary waves," IEEE J. Ocean. Eng. 22, 256-269.

Rouseff, D., Turgut, A., Wolf, S. N., Finette, S., Orr, M. H., Pasewark, B. H., Apel, J. R., Badiey, M., Chiu, C.-S., Headrick, R. H., Lynch, J. F., Kemp, J. N., Newhall, A. E., von der Heydt, K., and Tielbuerger, D. (2002). "Coherence of acoustic modes propagating through shallow water internal waves," J. Acoust. Soc. Am. 111, 1655-1666.

Zhou, J., Zhang, X., and Rogers, P. H. (1991). "Resonant interaction of sound wave with internal solitons in the coastal zone," J. Acoust. Soc. Am. 90, 2042-2054. 